\title{
The Prognostic Value of Pyrosequencing-Detected MGMT Promoter Hypermethylation in Newly Diagnosed Patients with Glioblastoma
}

\author{
Veronica Villani, ${ }^{1}$ Beatrice Casini, ${ }^{2}$ Andrea Pace, ${ }^{1}$ Luca Prosperini, ${ }^{3}$ \\ Carmine M. Carapella, ${ }^{4}$ Antonello Vidiri, ${ }^{5}$ Alessandra Fabi, ${ }^{6}$ and Mariantonia Carosi ${ }^{2}$ \\ ${ }^{1}$ Neuro-Oncology Unit, "Regina Elena" National Cancer Institute, Via Elio Chianesi 53, 00144 Rome, Italy \\ ${ }^{2}$ Division of Pathology, "Regina Elena" National Cancer Institute, Via Elio Chianesi 53, 00144 Rome, Italy \\ ${ }^{3}$ Department of Neurology and Psychiatry, Sapienza University, Viale dell'Università 30, 00185 Rome, Italy \\ ${ }^{4}$ Division of Neurosurgery, "Regina Elena" National Cancer Institute, Via Elio Chianesi 53, 00144 Rome, Italy \\ ${ }^{5}$ Service of Neuroradiology, "Regina Elena" National Cancer Institute, Via Elio Chianesi 53, 00144 Rome, Italy \\ ${ }^{6}$ Division of Medical Oncology, "Regina Elena” National Cancer Institute, Via Elio Chianesi 53, 00144 Rome, Italy \\ Correspondence should be addressed to Luca Prosperini; luca.prosperini@uniromal.it
}

Received 1 July 2015; Accepted 28 July 2015

Academic Editor: Stamatios Theocharis

Copyright (C) 2015 Veronica Villani et al. This is an open access article distributed under the Creative Commons Attribution License, which permits unrestricted use, distribution, and reproduction in any medium, provided the original work is properly cited.

\begin{abstract}
O6-methylguanine-DNA-methyltransferase (MGMT) has emerged as a relevant predictor of therapeutic response and good prognosis in patients with glioblastoma (GBM). Transcriptionally active MGMT rapidly removes the alkyl adducts, preventing the formation of cross-links and thereby causing resistance to alkylating drugs. Studies with pyrosequencing (PSQ) showed that this technique has a higher reproducibility and sensitivity than other techniques. However, the definition of a prognostically relevant threshold for the percentage of MGMT methylation remains one of the most critical issues in the use of PSQ analysis. The aim of this study was to define the cut-off value correlated with good favourable prognostic outcomes. We retrospectively analyzed 51 patients (33 males, 18 females) with GBM who underwent surgery or biopsy. The Receiver Operating Characteristics analysis showed that the best possible criteria for PSQ-detected percentage of MGMT methylation that predicted progression-free survival (PFS) and overall survival (OS) were $19 \%$ and $13 \%$, respectively. Patients with $\leq 19 \%$ of PSQ-detected MGMT had a shorter PFS (HR: 0.24 , $p<0.01$ ); those ones with $\leq 13 \%$ had a shorter OS (HR: $0.33, p<0.05$ ). Our study reinforces the importance of MGMT in the management of GBM patients, but future studies with larger sample sizes are warranted to confirm our findings.
\end{abstract}

\section{Introduction}

Glioblastoma multiforme (GBM) is the most frequent primary brain tumour in adults and is associated with poor prognosis [1]. The standard of care for patients with GBM currently involves surgical resection and temozolomide chemotherapy with concomitant radiotherapy, followed by cycles of adjuvant temozolomide [1]. Although some clinical trials have recently demonstrated that the standard treatment improves overall survival, only one-third of GBM patients seem to benefit from these therapies. Alkylating chemotherapeutic agents, such as temozolomide, induce cell death by forming cross-links between adjacent DNA strands through alkylation of the $\mathrm{O} 6$ position of guanine [2]. Transcriptionally active O6-methylguanine-DNA-methyltransferase (MGMT) rapidly removes the alkyl adducts, preventing the formation of cross-links, thereby causing resistance to alkylating drugs [3].

The loss of MGMT protein expression caused by hypermethylation of the MGMT promoter reduces the DNA repair activity of glioma cells, overcoming their resistance to alkylating agents $[2,4-6]$. Therefore, methylation of MGMT has become a clinically relevant predictor of response to treatment in glioma patients [7-10].

MGMT promoter hypermethylation is associated with longer progression-free and overall survival in patients 
who receive alkylating chemotherapy in association with radiotherapy [10]. MGMT methylation status is currently incorporated into a more refined classification system and applied in the clinical decision-making process, but there is no evidence yet about what is the most accurate diagnostic tool to estimate MGMT promoter hypermethylation. Most studies used methylation-specific polymerase chain reaction (MS-PCR) [8, 11-13]; however, one of the major drawbacks of this technique is its operator-dependent nature, mainly due to the sample reading subjectivity and lack of automation. Studies that compared various techniques for the assessment of MGMT methylation status showed that pyrosequencing (PSQ) shows a better prediction of survival, in addition to higher reproducibility and sensitivity with respect to other techniques $[12,14-16]$. However, only few studies have investigated which is the most accurate cut-off value that could represent methylated or unmethylated status. Consequently, the definition of a prognostically relevant threshold for the percentage of MGMT methylation remains one of the most critical issues in the use of PSQ analysis [17-21]. In clinical practice methylation is very important because it is considered a strong predictor of response to chemotherapy with alkylating agents and may help to drive personalized treatment strategies.

Starting from these assumptions, in this study we aimed to define the best cut-off value for PSQ-detected MGMT promoter hypermethylation which correlated with the most favourable prognostic outcomes.

\section{Materials and Methods}

2.1. Patients. Data of patients affected by newly diagnosed primary GBM who underwent surgery or biopsy and followed at the Neuro-Oncology Unit of Regina Elena National Cancer Institute were retrospectively analyzed. Tissue samples were matched with a comprehensive set of clinical data collected in the database of Neuro-Oncology Unit for each patient. The database includes demographic, clinical, and molecular data, as well as data on response to treatments and outcomes, including progression-free survival (PFS) and overall survival (OS), which was considered the main outcomes of this study. PFS was defined as the time elapsed from the first day of treatment and the date on which disease "progresses" or the date on which the patient dies. OS was defined as the time elapsed from first day of treatment and the date of death.

2.2. Tissue Analysis. Tissue samples were analyzed by means of PSQ for methylation status of MGMT assessment according to standardized procedure.

Genomic DNA was isolated from 4-5 paraffin sections of glioblastoma tissue. At least 1 slice was stained with hematoxylin and eosin to control the percentage of tumor cells. Tumor samples with at least $70 \%$ of tumor cells were considered, and DNA extraction was performed with QIAamp DNA FFPE tissue (Quiagen). The extracted DNA was subjected to polymerase chain reaction (PCR) amplification with a forward primer and a biotinylated reverse primer using the "MGMT PLUS" kit (Diatech pharmacogenetics) and the
"Rotor-Gene TM 6000" instrument. The PCR condition for MGMT gene was $95^{\circ} \mathrm{C}$ for 5 minutes, 45 cycles of $95^{\circ} \mathrm{C}$ for 30 seconds, $53^{\circ} \mathrm{C}$ for 30 seconds, and $72^{\circ} \mathrm{C}$ for 20 seconds, $72^{\circ} \mathrm{C}$ for 5 minutes, and then Green signal acquisition at $60^{\circ} \mathrm{C}$ for 20 seconds. During amplification, the uracil product by modification of cytosine unmethylated is converted in thymine while 5-methylcytosine remains as cytosine; therefore we can distinguish, in the sequence, methylated from unmethylated cytosine. We performed PSQ methylation assay to evaluate $10 \mathrm{CpG}$ sites in the following regions: chr 10: 131,265,507$131,265,556$ using sequencing primer of MGMT Kit Diatech (Pharmocogenetics, Jesi, Ancona, Italy). PSQ analysis was performed with PyroMarker CpG software 1.0.11 (Qiagen). The software gives a mean methylation value for each $10 \mathrm{CpG}$ site and the total mean of all $10 \mathrm{CpG}$ sites.

2.3. Statistical Analysis. Descriptive statistics were used to summarize pertinent study information. Categorical variables were reported as frequencies and percentage values, while continuous variables were reported as mean values and their relative standard deviation (SD), or median (range), as appropriate.

Since PSQ provided a percentage of MGMT methylation, the optimal cut-off value identifying methylated or unmethylated status was estimated by using the Receiver Operating Characteristics (ROC) curve analysis. Across various cut-off points, Youden's index maximised the differences between real-positive and false-positive subjects; thus, the optimal cut-off values predicting overall survival and progression-free survival were estimated.

To investigate the prognostic relevance of the PSQdetected MGMT promoter hypermethylation, univariate and multivariable Cox proportional hazards models were carried out by considering separately the overall survival and the progression-free survival as dependent variables. Outcome measures were evaluated after 1 year of follow-up. Gender, age at diagnosis, surgery (extent of removal/biopsy), radiotherapy (yes/no), and chemotherapy (yes/no) were inserted into the models as covariates and percentage of MGMT methylation was inserted as independent variable. Additional univariate and multivariable Cox proportional hazards models were built by replacing the percentage of MGMT methylation with a dichotomous variable derived from the afore described ROC analyses.

Lastly, since MGMT promoter hypermethylation is known to impact the response to treatment [17], Cox regression analyses were repeated only in the subsample who were treated with Stupp regimen [1].

All $p$ values less than 0.05 (two-sided) were considered as significant. Data were analysed with the Statistical Package for Social Sciences, version 21.0 (IBM SPSS, Chicago, IL, USA).

\section{Results}

3.1. Participants. In total, 51 patients ( 33 men, 18 women) diagnosed as affected by GBM from June 2013 to March 2015 were included in this study. Table 1 shows the characteristics of the study sample. The mean (SD) age of the patients was 61.7 (12.9) years. There were no significant differences 
TABLE 1: Demographic and clinical features of the study sample $(n=$ $51)$.

\begin{tabular}{|c|c|}
\hline Baseline characteris & \\
\hline Gender, $n(\%)$ & \\
\hline Women & $18(35 \%)$ \\
\hline Men & $33(65 \%)$ \\
\hline Age, years & \\
\hline Mean (SD) & $61.7(12.9)$ \\
\hline Median (range) & $62(25-84)$ \\
\hline MGMT methylatio & \\
\hline Mean (SD) & $21.5(19.1)$ \\
\hline Median (range) & $16(2-85)$ \\
\hline Treatment history & \\
\hline Surgery, $n(\%)$ & \\
\hline Done & $46(90 \%)$ \\
\hline Not done & $5(10 \%)$ \\
\hline Radiotherapy, $n$ (\% & \\
\hline Done & $39(76 \%)$ \\
\hline Not done & $12(24 \%)$ \\
\hline Chemotherapy, $n$ ( & \\
\hline Done & $41(80 \%)$ \\
\hline Not done & $10(20 \%)$ \\
\hline Main outcomes & \\
\hline Overall survival, $n$ & \\
\hline Dead & $24(47 \%)$ \\
\hline Alive & $27(53 \%)$ \\
\hline Progression-free su & \\
\hline Progressed & $34(67 \%)$ \\
\hline Not progressed & $17(23 \%)$ \\
\hline Follow-up time, m & \\
\hline Mean (SD) & $11.9(6.8)$ \\
\hline Median (range) & $12(3-27)$ \\
\hline
\end{tabular}

${ }^{*}$ Follow-up time refers to time from disease diagnosis and death or last visit.

between women and men in terms of baseline demographic and clinical characteristics. The mean and median percentage of MGMT methylation, as detected by PSQ, were $21.5 \%$ and $16 \%$ (ranging from 2 to 85 ). No significant relationships were observed between gender, age at diagnosis, time from disease onset to diagnosis and percentage of MGMT methylation.

3.2. Follow-Up Data. The median follow-up time was 12 months (ranging from 3 to 27 months).

PFS: thirty-four (64\%) patients experienced disease progression after a mean time of 12.1 (5.6) months from the diagnosis (ranging from 4 to 18 ).

OS: twenty-four (47\%) patients died after a mean followup time of 12.6 (7.2) months from the diagnosis (ranging from 3 to 27 ), while the mean follow-up time from the diagnosis to last visit was 11.3 (6.5) months for the 27 survivors (53\%) (ranging from 3 to 26$)(p=0.5)$.

3.3. Receiver Operating Characteristics Analyses. The main findings of the ROC analyses are summarized in Table 2. The best possible criteria for PSQ-detected percentage of
TABLE 2: Receiver Operating Characteristic (ROC) analyses showing the best cut-off values of MGMT predicting overall survival (left) and progression-free survival (right) in the study sample $(n=51)$.

\begin{tabular}{lcc}
\hline & $\begin{array}{c}\text { Progression-free } \\
\text { survival }\end{array}$ & Overall survival \\
\hline AUC (95\% CIs) & $0.71(0.57-0.83)$ & $0.65(0.50-0.78)$ \\
Criterion (cut-off) & $\geq 19 \%$ & $\geq 13 \%$ \\
Sensitivity & $73 \%$ & $58 \%$ \\
Specificity & $77 \%$ & $70 \%$ \\
$p$ value & $\mathbf{0 . 0 0 5 4}$ & 0.057 \\
\hline
\end{tabular}

AUC: area under the curve; MGMT: O6-methylguanine-DNAmethyltransferase.

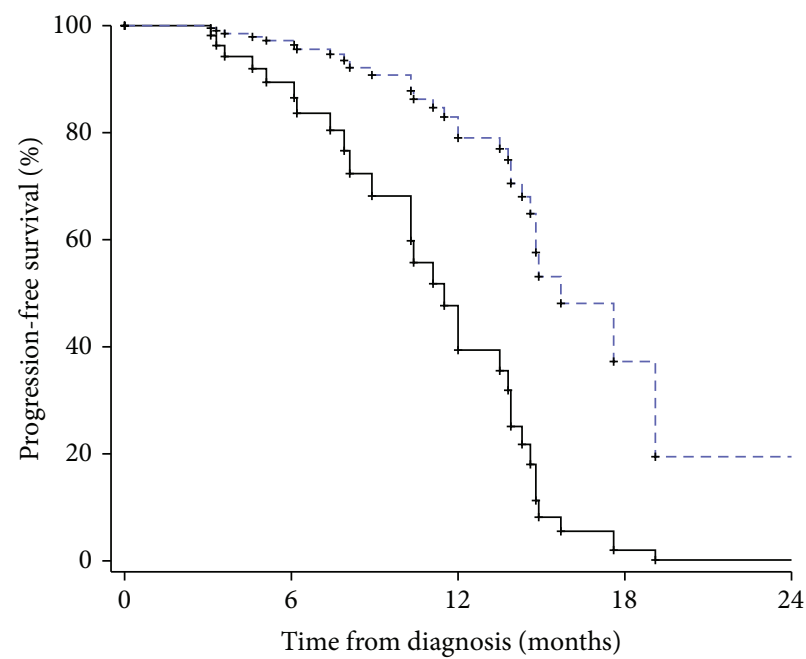

FIGURE 1: Kaplan-Meier curve showing the time to progression according to the percentage of MGMT methylation (<19\%: continue line; $\geq 19 \%$ dotted line).

MGMT methylation that predicted PFS and OS were $19 \%$ and $13 \%$, respectively. Adopting a cut-off value of $19 \%$ of MGMT methylation ensured a sensitivity of $73 \%$ and specificity of $77 \%$ in predicting progression-free survival $(p=0.0054)$. Adopting a cut-off value of $13 \%$ of MGMT methylation ensured a sensitivity of $58 \%$ and specificity of $70 \%$ in predicting overall survival $(p=0.057)$.

3.4. Time-to-Event Analyses. Kaplan-Meier curves show that patients who had less than $19 \%$ and 13\% of MGMT methylation experienced worse PFS and OS, respectively (both $p$ values $<0.05$ by the Log-Rank test; see also Figures 1 and 2 ).

In the final Cox models, the variables which resulted in predicting the PFS and OS were those shown in Tables 3 and 4 , respectively.

The risk of disease progression was reduced by $3 \%$ for each unit of increase in percentage of MGMT methylation (HR: $0.97,95 \%$ CIs $0.94-0.99, p<0.01$ ). Consistently, patients who presented more than 19\% MGMT methylation had a 76\%-decreased risk of disease progression (HR: 0.24, 95\% CIs 0.10-0.64, $p<0.01$ ). 
TABLE 3: Cox proportional hazard models predicting progression-free survival (dependent variable).

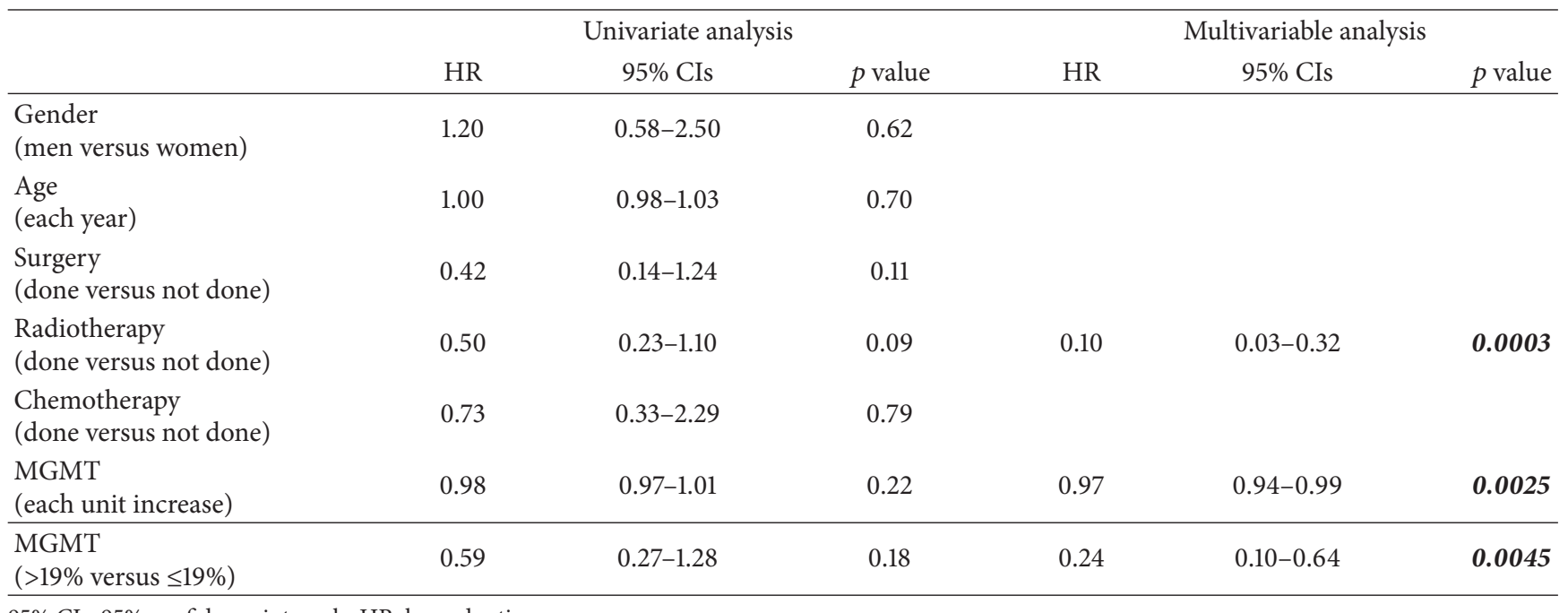

95\% CIs: 95\% confidence intervals; HR: hazard ratio.

TABLE 4: Cox proportional hazard models predicting overall survival (dependent variable).

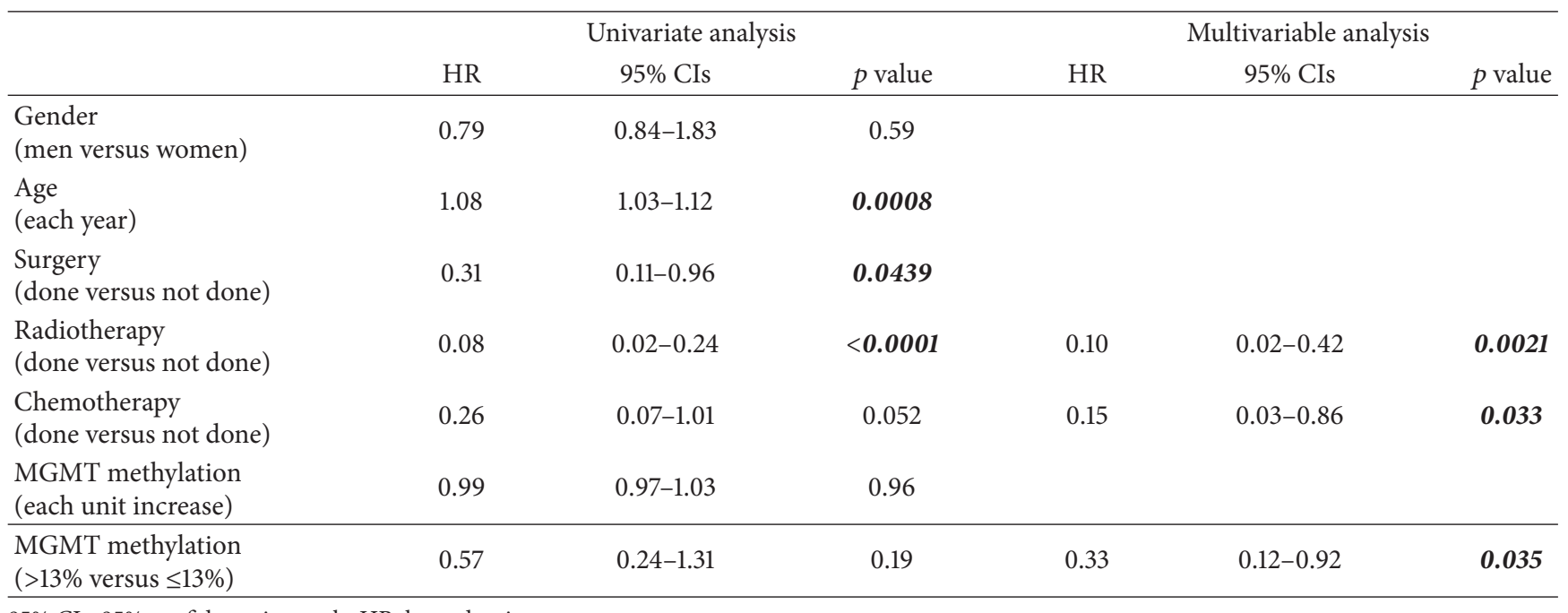

95\% CIs: 95\% confidence intervals; HR: hazard ratio.

While the percentage of MGMT methylation did not contribute to fitting the multivariable analysis predicting OS (and thereby was excluded from the model), those patients who presented a MGMT methylation above $13 \%$ had a $67 \%$ reduced risk of death (HR: $0.33,95 \%$ CIs $0.12-0.92, p<0.05$ ).

3.5. Additional Analysis on Patients Treated with Stupp Regimen. A total of 32 patients (63\% of the whole study sample) were treated with Stupp regimen and were followed up to a median time of 15 months (ranging from 4 to 27 months). Despite the reduced size of this subsample, more than $19 \%$ of MGMT methylation was still predictive of the risk of disease progression (HR: 0.16, 95\% CIs 0.03-0.73, $p<0.05$ ). By contrast, the cut-off value of $13 \%$ of MGMT methylation did not predict OS in the subgroup of patients treated with Stupp regimen (HR: $0.35,95 \%$ CIs $0.10-1.27, p=0.1$ ), probably due to the small sample size.

\section{Discussion}

MGMT methylation status is considered an important marker for the prognosis and therapeutic response of patients with newly diagnosed GBM who are treated with standard care [1]. However, there are still some open questions, mainly concerning what is the best technique for the assessment of methylation and what is the optimal threshold indicating methylated or unmethylated status. Recent studies have reported that the best predictive value was obtained by PSQ compared to other techniques [12], but cut-off values for the percentage of methylation that define the methylation status remain one of the most critical issues. PSQ allows highly reproducible quantitative evaluation of methylation at discrete $\mathrm{CpG}$ sites therapy providing more information on promoter methylation patterns. Locus-specific hypermethylation, mostly at the $\mathrm{CpG}$ island (CGI) promoters, is frequent 


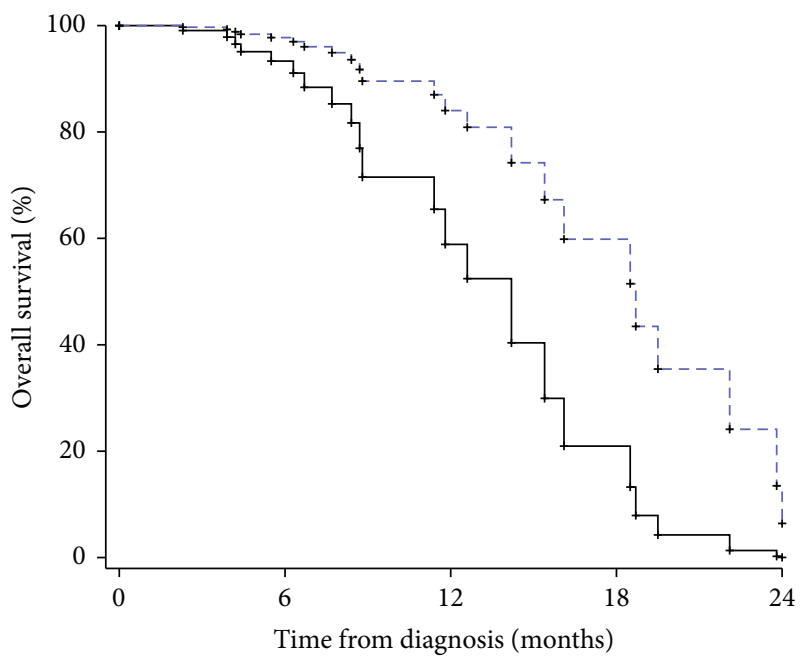

FIGURE 2: Kaplan-Meier curve showing time to death according to the percentage of MGMT methylation $(<13 \%$ : continue line; $\geq 13 \%$ dotted line).

in patients with GBM. CGIs are regions of about $500 \mathrm{bp}$ to $1 \mathrm{~kb}$ in which CpG nucleotides are approximately five times more abundant compared to the rest of the genome. MGMT encodes a DNA repair protein that removes alkyl adducts at the $\mathrm{O} 6$ position of guanine. MGMT expression protects normal cells from carcinogens; however, it can also protect cancer cells from chemotherapeutic alkylating agents [18]. Therefore, MGMT status is a strong predictor of response to treatment with temozolomide and it is determined in most ongoing clinical trials using this drug $[1,7,8]$.

Most studies reporting a link between MGMT status and survival in patients with GBM have used PSQ [17-21]. Findings from the present study confirm that the percentages of methylation estimated by PSQ are highly correlated with prognostic outcome in these patients. In the present study, the best possible criteria for PSQ-detected percentage of MGMT methylation that predicted PFS and OS were 19\% and 13\%, respectively. These data slightly deviate from literature data that reported lower cut-off values, ranging from $8 \%$ to $10 \%$ $[17,20,22]$. Dunn et al. [17] considered methylated those patients who had $\geq 9 \%$ average methylation and unmethylated those ones who had an average methylation $<9 \%$ in all samples. Mikeska et al. [20] reported that unmethylated tumor samples and control samples showed ratios of $<10 \%$ at all positions with a small SD and suggested a score to separate unmethylated and methylated cases by employing the percentage values of four specified CpGs. Wiewrodt et al. [22] showed that patients expressing $\leq 30$ fmolmg-1 MGMT protein in the pretreatment tumor volume had a significantly better response to alkylating therapy than those with MGMT protein above this level.

However, consistently with literature data, we observed that PSQ had good sensitivity and specificity ( $73 \%$ and $77 \%$, resp.) in predicting PFS ( $p=0.0054)$, while its sensitivity decreases to $58 \%$, and specificity remains good $(70 \%)$, in predicting OS $(p=0.057)$.
In our study, the risk of disease progression was reduced by $3 \%$ for each unit increase in percentage of MGMT methylation (HR: 0.97, 95\% CIs 0.94-0.99, $p<0.01$ ). Consistently, patients who presented more than 19\% MGMT methylation had a $76 \%$-decreased risk of disease progression (HR: $0.24,95 \%$ CIs $0.10-0.64, p<0.01$ ). Also patients who presented a MGMT methylation above $13 \%$ had a $67 \%$ reduced risk of death (HR: 0.33, 95\% CIs 0.12-0.92, $p<0.05$ ). The additional analysis only on patients treated with Stupp regimen [1] strengthens our findings and reinforces the role of MGMT methylation in predicting the response to treatment, at least in terms of PFS [17].

Taken together, these results support the hypothesis that MGMT methylation is a relevant prognostic marker since it impacts on disease progression and survival. For this reason, we strongly recommend molecular assay for the assessment of MGMT status in the management of all patients affected by newly diagnosed GBM. The main limit of the present study is the small sample size. For this reason, future studies on larger population are warranted to confirm the cut-off values we have found as the best possible predictive criteria of good prognosis.

\section{Conflict of Interests}

The authors declare that there is no conflict of interests regarding the publication of this paper.

\section{Acknowledgment}

The authors would like to thank Ms. Tania Merlino for the English language assistance.

\section{References}

[1] R. Stupp, W. P. Mason, M. J. van den Bent et al., "Radiotherapy plus concomitant and adjuvant temozolomide for glioblastoma," The New England Journal of Medicine, vol. 352, pp. 987996, 2005.

[2] M. E. Hegi, L. Liu, J. G. Herman et al., "Correlation of O6methylguanine methyltransferase (MGMT) promoter methylation with clinical outcomes in glioblastoma and clinical strategies to modulate MGMT activity," Journal of Clinical Oncology, vol. 26, no. 25, pp. 4189-4199, 2008.

[3] B. Verbeek, T. D. Southgate, D. E. Gilham, and G. P. Margison, "O6-Methylguanine-DNA methyltransferase inactivation and chemotherapy," British Medical Bulletin, vol. 85, pp. 17-33, 2008.

[4] S. L. Gerson, "Clinical relevance of MGMT in the treatment of cancer," Journal of Clinical Oncology, vol. 20, no. 9, pp. 23882399, 2002.

[5] M. Esteller, J. Garcia-Foncillas, E. Andion et al., "Inactivation of the DNA-repair gene MGMT and the clinical response of gliomas to alkylating agents," The New England Journal of Medicine, vol. 343, pp. 1350-1354, 2000.

[6] M. E. Hegi, A. C. Diserens, S. Godard et al., "Clinical trial substantiates the predictive value of O-6-methylguanineDNA methyltransferase promoter methylation in glioblastoma patients treated with temozolomide," Clinical Cancer Research, vol. 10, pp. 1871-1874, 2004. 
[7] M. E. Hegi, A. C. Diserens, T. Gorlia et al., "MGMT gene silencing and benefit from temozolomide in glioblastoma," The New England Journal of Medicine, vol. 352, no. 10, pp. 997-1003, 2005.

[8] M. Weller, R. Stupp, G. Reifenberger et al., "MGMT promoter methylation in malignant gliomas: ready for personalized medicine?" Nature Reviews Neurology, vol. 6, no. 1, pp. 39-51, 2009.

[9] A. L. Rivera, C. E. Pelloski, M. R. Gilbert et al., "MGMT promoter methylation is predictive of response to radiotherapy and prognostic in the absence of adjuvant alkylating chemotherapy for glioblastoma," Neuro-Oncology, vol. 12, no. 2, pp. 116-121, 2010.

[10] J. Felsberg, M. Rapp, S. Loeser et al., "Prognostic significance of molecular markers and extent of resection in primary glioblastoma patients," Clinical Cancer Research, vol. 15, no. 21, pp. 6683-6693, 2009.

[11] M. Weller, J. Felsberg, C. Hartmann et al., "Molecular predictors of progression-free and overall survival in patients with newly diagnosed glioblastoma: a prospective translational study of the German Glioma Network," Journal of Clinical Oncology, vol. 27, no. 34, pp. 5743-5750, 2009.

[12] L. Karayan-Tapon, V. Quillien, J. Guilhot et al., "Prognostic value of O6-methylguanine-DNA methyltransferase status in glioblastoma patients, assessed by five different methods," Journal of Neuro-Oncology, vol. 97, no. 3, pp. 311-322, 2010.

[13] P. Metellus, B. Coulibaly, I. Nanni et al., "Prognostic impact of O6-methylguanine-DNA methyltransferase silencing in patients with recurrent glioblastoma multiforme who undergo surgery and carmustine wafer implantation: a prospective patient cohort," Cancer, vol. 115, no. 20, pp. 4783-4794, 2009.

[14] I. Vlassenbroeck, S. Califice, A. C. Diserens et al., "Validation of real-time methylation-specific PCR to determine $O^{6}$ methylguanine-DNA methyltransferase gene promoter methylation in glioma," The Journal of Molecular Diagnostics, vol. 10, no. 4, pp. 332-337, 2008.

[15] V. Quillien, A. Lavenu, L. Karayan-Tapon et al., "Comparative assessment of 5 methods (methylation-specific polymerase chain reaction, MethyLight, pyrosequencing, methylationsensitive high-resolution melting, and immunohistochemistry) to analyze O6-methylguanine-DNA-methyltranferase in a series of 100 glioblastoma patients," Cancer, vol. 118, no. 17, pp. 4201-4211, 2012.

[16] A. B. Håvik, P. Brandal, H. Honne et al., "MGMT promoter methylation in gliomas-assessment by pyrosequencing and quantitative methylation-specific PCR," Journal of Translational Medicine, vol. 10, article 36, 2012.

[17] J. Dunn, A. Baborie, F. Alam et al., "Extent of MGMT promoter methylation correlates with outcome in glioblastomas given temozolomide and radiotherapy," British Journal of Cancer, vol. 101, no. 1, pp. 124-131, 2009.

[18] V. Quillien, A. Lavenu, M. Sanson et al., "Outcome-based determination of optimal pyrosequencing assay for MGMT methylation detection in glioblastoma patients," Journal of Neuro-Oncology, vol. 116, no. 3, pp. 487-496, 2014.

[19] M. Uno, S. M. Oba-Shinjo, A. A. Camargo et al., "Correlation of MGMT promoter methylation status with gene and protein expression levels in glioblastoma," Clinics, vol. 66, no. 10, pp. 1747-1755, 2011.

[20] T. Mikeska, C. Bock, O. El-Maarri et al., "Optimization of quantitative MGMT promoter methylation analysis using pyrosequencing and combined bisulfite restriction analysis,"
The Journal of Molecular Diagnostics, vol. 9, no. 3, pp. 368-381, 2007.

[21] H. Xie, R. Tubbs, and B. Yang, "Detection of MGMT promoter methylation in glioblastoma using pyrosequencing," International Journal of Clinical and Experimental Pathology, vol. 8, pp. 636-642, 2015.

[22] D. Wiewrodt, G. Nagel, N. Dreimüller, T. Hundsberger, A. Perneczky, and B. Kaina, "MGMT in primary and recurrent human glioblastomas after radiation and chemotherapy and comparison with p53 status and clinical outcome," International Journal of Cancer, vol. 122, no. 6, pp. 1391-1399, 2008. 


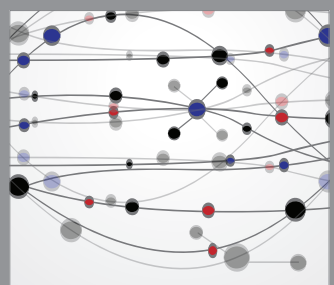

The Scientific World Journal
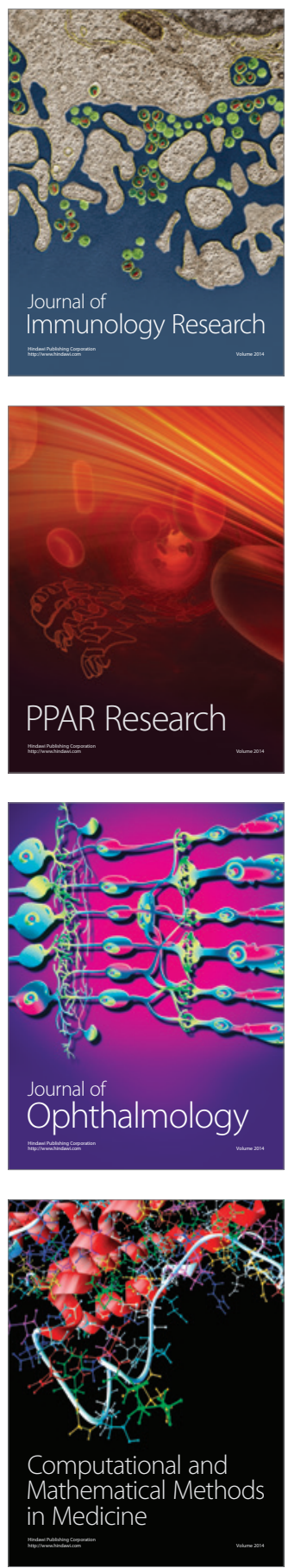

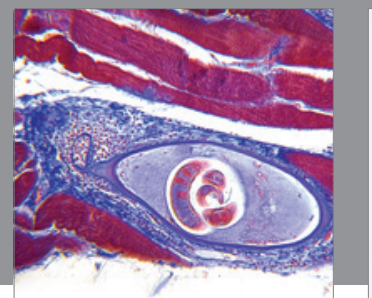

Gastroenterology

Research and Practice
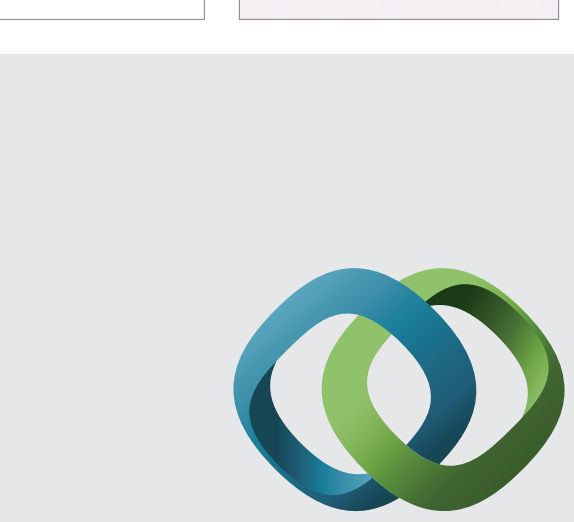

\section{Hindawi}

Submit your manuscripts at

http://www.hindawi.com
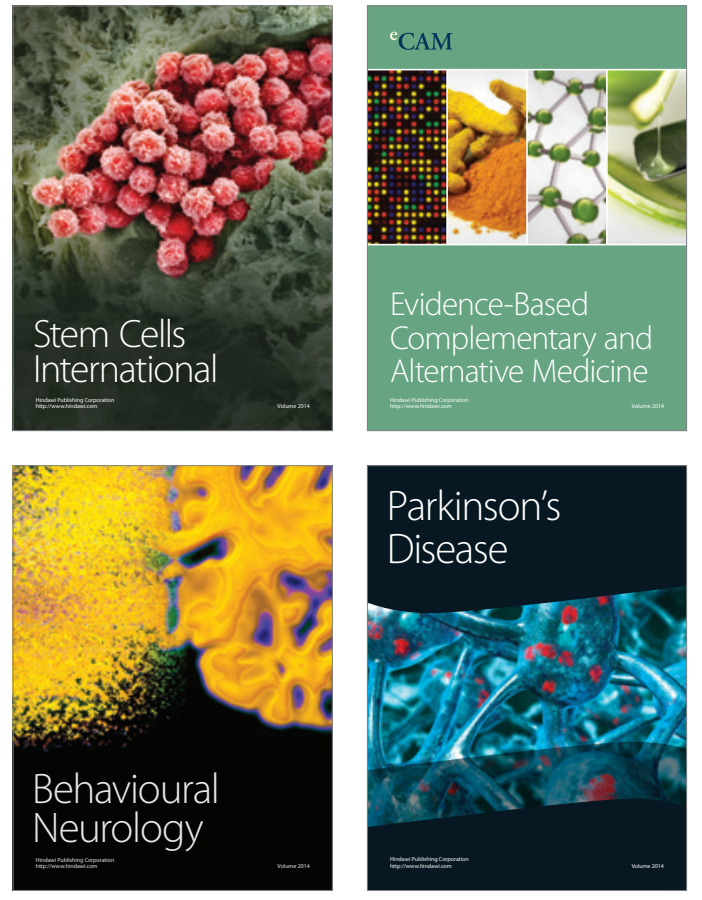
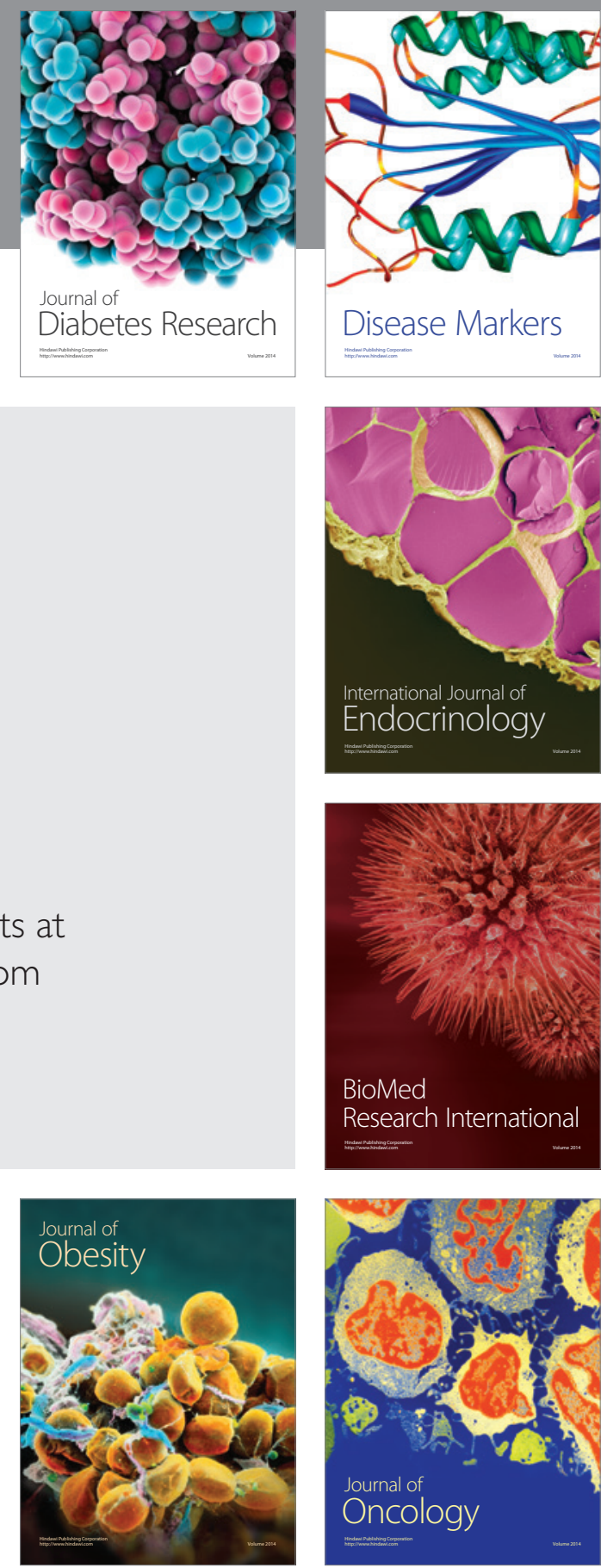

Disease Markers
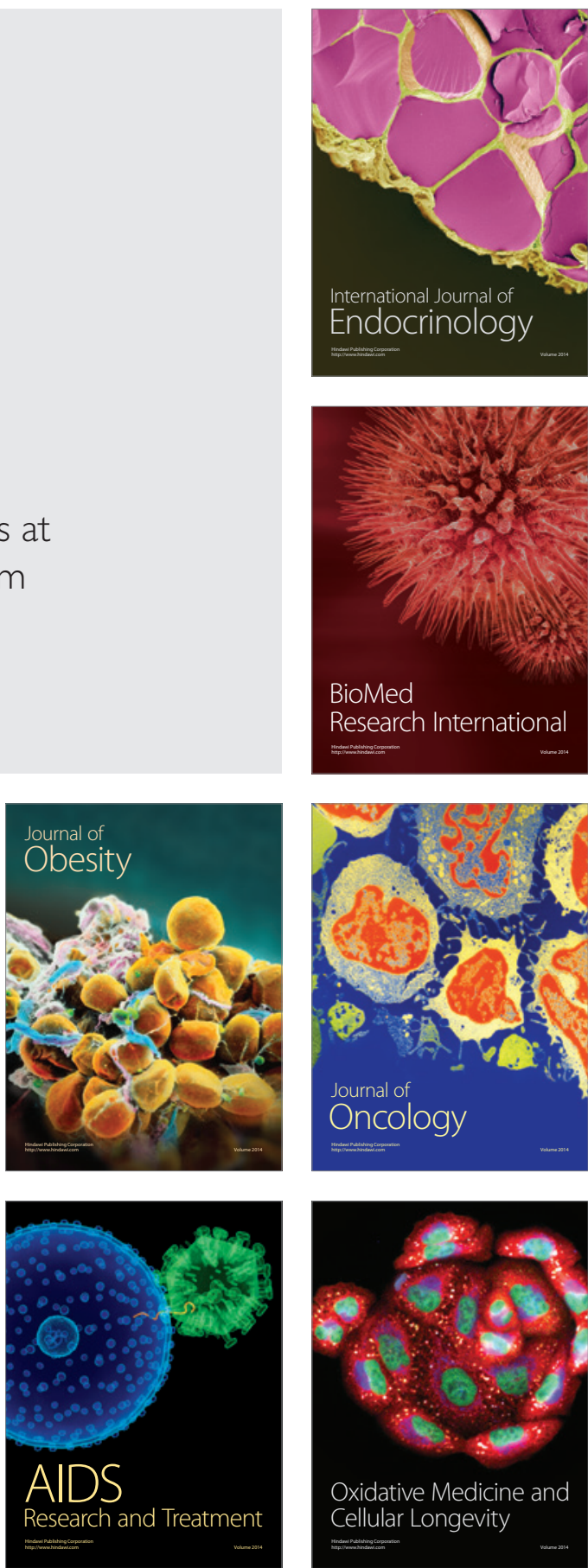